\title{
Sterile neutrinos as a possible explanation for the upward air shower events at ANITA
}

\author{
Guo-yuan Huang* \\ Institute of High Energy Physics, Chinese Academy of Sciences, Beijing 100049, China \\ and School of Physical Sciences, University of Chinese Academy of Sciences, \\ Beijing 100049, China
}

(Received 7 July 2018; published 24 August 2018)

\begin{abstract}
The ANITA experiment has observed two unusual upgoing air shower events, which are consistent with the $\tau$-lepton decay origin. However, these events are in contradiction with the standard neutrino-matter interaction models as well as the EeV diffuse neutrino flux limits set by IceCube and the cosmic ray facilities like AUGER. In this paper, we have reinvestigated the possibility of using the sterile neutrino hypothesis to explain the ANITA anomalous events. The diffuse flux of the sterile neutrinos is less constrained by the IceCube and AUGER experiments due to the small active-sterile mixing angle suppression. The quantum decoherence effect should be included for describing the neutrino flux propagating in the Earth matter, because the interactions between neutrinos and the Earth matter are very strong at the EeV energy scale. After several experimental approximations, we show that the ANITA anomaly itself is able to be explained by the sterile neutrino origin, but we also predict that the IceCube observatory should have more events than ANITA. It makes the sterile neutrino origin very unlikely to account for both of them simultaneously. A more solid conclusion can be drawn by the dedicated ANITA signal simulation.
\end{abstract}

DOI: $10.1103 /$ PhysRevD.98.043019

\section{INTRODUCTION}

The cosmic rays with an energy around the GreisenZatsepin-Kuzmin (GZK) cutoff $(\sim 50 \mathrm{EeV})$ would be strongly suppressed due to the interactions with the cosmic microwave background [1,2], i.e., $p+\gamma_{\mathrm{CMB}} \rightarrow p($ or $n)+$ $\mathbf{n} \pi, p+\gamma_{\mathrm{CMB}} \rightarrow \Delta^{+}(1232) \rightarrow p+\pi^{0}\left(\right.$ or $\left.n+\pi^{+}\right)$, where $\mathbf{n}$ is the total number of the produced $\pi$ 's. The ultrahigh energy (UHE) neutrinos at the scale of $\mathrm{EeV}$ could be copiously produced by the subsequent decays of secondary charged pions and neutrons [3]. The EeV neutrino remains to be detected, and the Antarctic Impulsive Transient Antenna (ANITA) experiment [4] is dedicated to the detection of these cosmogenic neutrinos.

In 2016, the ANITA experiment has reported one unusual steeply upward-pointing cosmic ray event 3985267 with a shower energy around $0.6 \mathrm{EeV}$ during the ANITA-I flight [5]. This shower event could be generated by the decay of a $\tau$ lepton, which is emerging from the surface of the ice with the zenith angle around

\footnotetext{
*huanggy@ihep.ac.cn
}

Published by the American Physical Society under the terms of the Creative Commons Attribution 4.0 International license. Further distribution of this work must maintain attribution to the author(s) and the published article's title, journal citation, and DOI. Funded by SCOAP ${ }^{3}$. $63^{\circ}$, and the $\tau$ lepton should be interpreted as the product of a parent $\nu_{\tau}$ by the charged-current (CC) interactions with the Earth matter. However, such a hypothesis is strongly disfavored due to the fact that the Earth $\mathrm{CC}$ attenuation coefficient is $4 \times 10^{-6}$ for the neutrinos coming from such a steep angle [5]. The associated event number around $E_{\nu} \sim 1 \mathrm{EeV}$ is negligible after adopting the IceCube bound on the diffuse $\mathrm{EeV}$ neutrinos [6,7], approximately $E_{\nu}^{2} \mathrm{~d} \Phi_{\nu} /$ $\mathrm{d} E_{\nu} \lesssim 2 \times 10^{-8} \mathrm{GeV} \cdot \mathrm{cm}^{-2} \mathrm{~s}^{-1} \mathrm{sr}^{-1}$. In addition, there should be more Earth-skimming events than the steep events. The situation is worse after the ANITA detector observed the second such air shower event 15717147 with an energy around $0.56 \mathrm{EeV}$ at an even steeper zenith angle $\sim 55^{\circ}$ during the ANITA-III flight [8].

Possible explanations for the anomalous events including the large transient point-source flux [8], the transition radiation of the Earth-skimming neutrinos [9], the sterile neutrino origin [10], and the decay of the quasistable dark matter in the Earth's core [11] have been investigated in the literature. After the report of the first anomalous event, Ref. [10] has proposed that the sterile neutrinos could be the origin of such an event. The sterile neutrinos are well

\footnotetext{
${ }^{1}$ The reported emergence angle of the event 3985267 is $\sim 27^{\circ}$ below the horizontal; the corresponding zenith angle is therefore $\sim 63^{\circ}$. One should be aware that the ANITA horizon is around $6^{\circ}$ below; it is horizontal because of its altitude.
} 
motivated by several particle physics issues and experimental anomalies. The heavy sterile neutrinos can explain the mass generation of light neutrinos through the seesaw mechanism [12-15], the sterile neutrino in the $\mathrm{keV}$ mass range is a good candidate of the warm dark matter [16], and the anomalies of the short baseline experiments like LSND and MiniBOONE; the Gallium source experiments as well as the reactor neutrino experiments hint at the existence of the eV-scale sterile neutrinos [17-22]. To explain the ANITA anomalous events, we need a strong sterile neutrino flux. The sources of the flux could be the superheavy dark matter decay [23-31], the topological defects [28], or some exotic interaction [32-38], which converts active neutrinos into sterile neutrinos during their propagation. When the sterile flux goes through the Earth, they will effectively experience a suppressed cross section due to the small active-sterile mixing. In such a way, the neutrinos can make their way to the thin interaction region below the ANITA detector, finally being converted to the $\tau$ lepton by the CC interaction with the ice, water, or rock. However, according to the analysis of Ref. [10], the sterile origin is in mild tension with the steep emergence angle, e.g., only $10 \%$ of the events are expected to emerge with the zenith angle smaller than $63^{\circ}$ for an active-sterile mixing angle $\theta=0.1$. Obviously, the second event 15717147 reported later [8] with a zenith angle $55^{\circ}$ increases the tension.

In our work, the sterile neutrino origin is reexamined, and we mainly have two treatments different from Ref. [10] which are addressed as follows:

(i) The neutrinos will lose coherence when strongly interacting with the ambient matter. After the flux of a sterile neutrino mass eigenstate enters the Earth, the matter frequently measures the neutrino states such that the survived flux will collapse into the sterile state $\nu_{\mathrm{s}}$.

(ii) Because a positive detection is made when the payload of ANITA is covered by the induced impulse cone with an angle around $1^{\circ}$ [8], only a very small fraction of the plane flux from each direction can be detected. Thus, the effective area $A_{\text {eff }}(\Omega)$ should be much smaller than the expectation of Ref. [10].

This work is organized as follows. In Sec. II, we investigate the evolution of the sterile neutrinos propagating in matter with the decoherence effect included, then the angular dependence is studied. In Sec. III, we give our predictions of the ANITA events for different sterile neutrino parameters based on several assumptions and approximations of the experimental setup. In Sec. IV, we make our conclusion.

\section{PROPAGATION OF STERILE NEUTRINOS}

The EeV sterile neutrinos will lose their coherence after traveling the galactic distance to the Earth. Under the twoflavor scheme, the initial neutrino fluxes are the mass eigenstates $\nu_{4}$ and $\nu_{1}$ with fractions of $\cos ^{2} \theta$ and $\sin ^{2} \theta$, respectively, where $\theta$ is the active-sterile mixing angle, $\nu_{1}$ harmlessly represents the three active neutrino mass eigenstates, and $\nu_{4}$ is the heavy neutrino mass eigenstate. When the $\nu_{4}$ flux propagates into the Earth, the Earth matter will frequently interact with, or measure, the neutrino's flavor. The $\nu_{4}$ state is the superposition of the active and sterile components, i.e., $\nu_{4}=\sin \theta \nu_{\mathrm{a}}+\cos \theta \nu_{\mathrm{s}}$, and only the active component $\nu_{\mathrm{a}}{ }^{2}$ is able to collide with the ambient matter through the $\mathrm{CC}$ or neutral current (NC) interactions. The Earth matter is like the quantum discriminator which can resolve the neutrino mixing, making the $\nu_{4}$ state to collapse into either a $\nu_{\mathrm{a}}$ state or $\nu_{\mathrm{s}}$ state; we refer the reader to [40-42] for more details.

We focus on the two-flavor scheme with $\nu_{\tau}$ and $\nu_{\mathrm{s}}$. It is the decay of a $\tau$ lepton that is responsible for the anomalous events of ANITA, while the electron and the muon are undetectable for ANITA. The realistic four-flavor scheme is quite complicated. The mixings among the three active neutrinos through the light mass eigenstates $\left(\nu_{1}, \nu_{2}\right.$, and $\left.\nu_{3}\right)$ are either heavily suppressed by the strong matter effect at the EeV energy, or becoming unimportant because the oscillation length $\left(\sim 10^{12} \mathrm{~km}\right.$ for EeV energy) is much longer than the Earth diameter [43]. The appearance probability from the active flavor to another active one $P\left(\nu_{\mathrm{a}}^{\prime} \leftrightarrow \nu_{\mathrm{a}}\right)$ should be proportional to $\sin ^{4} \theta$ instead of $\sin ^{2} \theta$ for $P\left(\nu_{\mathrm{s}} \leftrightarrow \nu_{\mathrm{a}}\right)$. The dominant mixing is between $\nu_{\mathrm{s}}$ and $\nu_{\mathrm{a}}$ for $\theta \ll 1$, and the $\nu_{\mathrm{a}}$-nucleon cross sections are almost same for the three active flavors. If we switch on the mixings $\nu_{\mathrm{s}}-\nu_{e}$ and $\nu_{\mathrm{s}}-\nu_{\mu}$, not only $\nu_{\tau}$ but also the $\nu_{e}$ and $\nu_{\mu}$ components can be generated by the oscillation of $\nu_{\mathrm{S}}$. However, the $\nu_{e}$ and $\nu_{\mu}$ fluxes are undetectable at ANITA but detectable for IceCube. For simplicity, we assume the mixings of $\nu_{\mathrm{s}}-\nu_{e}$ and $\nu_{\mathrm{s}}-\nu_{\mu}$ are negligible. To properly take the decoherence effect into account, we adopt the following evolution equation:

$$
\begin{aligned}
i \frac{\mathrm{d}}{\mathrm{d} t}\left(\begin{array}{c}
c_{\tau} \\
c_{\mathrm{s}}
\end{array}\right)= & \frac{1}{2 E_{\nu}}\left[U\left(\begin{array}{cc}
m_{1}^{2} & 0 \\
0 & m_{4}^{2}
\end{array}\right) U^{\dagger}+\left(\begin{array}{cc}
A_{\mathrm{NC}} & 0 \\
0 & 0
\end{array}\right)\right. \\
& \left.-i\left(\begin{array}{cc}
E_{\nu} / L_{\mathrm{atten}} & 0 \\
0 & 0
\end{array}\right)\right]\left(\begin{array}{l}
c_{\tau} \\
c_{\mathrm{s}}
\end{array}\right)
\end{aligned}
$$

for $\nu_{4}(t)=c_{\tau} \nu_{\tau}+c_{\mathrm{s}} \nu_{\mathrm{s}}$, where $U$ is the $2 \times 2$ active-sterile mixing matrix with the mixing angle $\theta, m_{1}$ is the averaged active neutrino mass which is negligible compared with $m_{4}$ at the keV scale, $A_{\mathrm{NC}}=-G_{\mathrm{F}}\left(1-Y_{e}\right) n_{\mathrm{N}} / \sqrt{2}$ represents the NC matter effect with $G_{\mathrm{F}}$ being the Fermi constant, $Y_{e}$ the fraction of electrons and $n_{\mathrm{N}}$ the nucleon number density of the matter, and $L_{\text {atten }}$ is the local attenuation length of the neutrino. $L_{\text {atten }}$ depends on the nucleon density and the neutrino energy with the relation $L_{\text {atten }}=\left[\sigma\left(E_{\nu}\right) n_{\mathrm{N}}\right]^{-1}$. The number density profile of the Earth can be found in the PREM model [44]. The NC and CC cross sections are referred to [45], and we remark that both the $\mathrm{CC}$ and $\mathrm{NC}$

\footnotetext{
${ }^{2}$ See [39] and the references therein for recent constraints on the active-sterile neutrino mixing.
} 
interactions contribute to the attenuation effect. We have neglected the usual subleading regeneration effects for simplicity. ${ }^{3}$ The initial conditions for the evolution read $c_{\tau}(0)=\sin \theta, c_{\mathrm{s}}(0)=\cos \theta$ before the $\nu_{4}$ flux enters the Earth.

Before turning to numerical demonstration of the evolution, we can first have some analytical observations on Eq. (1). If we ignore the oscillation terms, i.e., the first two terms in the right-hand side of Eq. (1), the evolution is trivial. The active and sterile components evolve independently such that the active component is quickly absorbed by the Earth with only the unobservable sterile component left, and there will be null signal in the detector as in the standard case. However, the sterile and active neutrinos are assumed to be mixed and can oscillate from one to the other if the propagation length covers the oscillation length of $L_{\mathrm{osc}} \equiv 4 \pi E_{\nu} / \Delta m_{41}^{2} \approx 2476 \mathrm{~km}\left[E_{\nu} /(1 \mathrm{EeV})\right]\left[(1 \mathrm{keV})^{2} /\right.$ $\left.\Delta m_{41}^{2}\right]$. For the ANITA events 3985267 and 15717147 with emitting zenith angles of $63^{\circ}$ and $55^{\circ}$, the corresponding chord lengths are $5785 \mathrm{~km}$ and $7309 \mathrm{~km}$, respectively, assuming a spherical Earth structure. Therefore, it is quite evident that the mass of $\nu_{4}$ should be around the $\mathrm{keV}$ scale or even larger to convert the $\nu_{\mathrm{S}}$ flux into the $\nu_{\tau}$ flux when traversing the Earth. We show in Fig. 1, a simple sketch of the scenario. In such a way, the $\nu_{\tau}$ flux can be regenerated and survive the attenuation of the Earth.

For the keV-sterile neutrinos, the Mikheyev-SmirnovWolfenstein (MSW) resonance condition inside the Earth can be fulfilled. However, the associated total flavor conversion effect at the resonance point as in the discussion of the solar neutrino problem does not work here. The matter term in the Earth mantle for the EeV neutrino reads $A_{\mathrm{NC}} \approx 0.1 \mathrm{keV}^{2}$. To satisfy the resonance condition $A_{\mathrm{NC}}=$ $\Delta m_{41}^{2} \cos \theta$ for the antineutrinos with the mixing angle $\theta$, the $m_{4}$ should be around $0.3 \mathrm{keV}[\sqrt{\cos \theta}]^{-1}$. The effective mass-squared difference when the resonance is achieved reads $\Delta \tilde{m}_{41}^{2}=\Delta m_{41}^{2} \sin 2 \theta \approx 0.2 \sin \theta \mathrm{keV}^{2}$, which corresponds to an oscillation length of $[12370 / \sin \theta] \mathrm{km} \sim$ $R / \sin \theta$ with $R=12742 \mathrm{~km}$ being the diameter of the Earth. Note that the density of the Earth varies very rapidly, by $20 \%$ for the Earth mantle of $2000 \mathrm{~km}$. It is very unlikely for those neutrinos to stay around the resonance while developing the phase. Our numerical calculation has included the matter effect without making any approximations. For a realistic Earth density model, the castlelike density structure can possibly induce the parametric resonance effect, i.e., the probability drifts with the periodically fluctuating matter density along the neutrino trajectory. For a neutrino beam traversing the Earth, the period of matter density profile is $3 / 2$. To satisfy the parametric resonance condition, the phase developing process must keep undisturbed along the trajectory. However, due

\footnotetext{
${ }^{3}$ The $\tau$ lepton produced by the CC can decay back to $\nu_{\tau}$. For the $\mathrm{NC}$ interaction, the daughter neutrino carries averagely $80 \%$ of the initial energy, not simply removed from the flux.
}

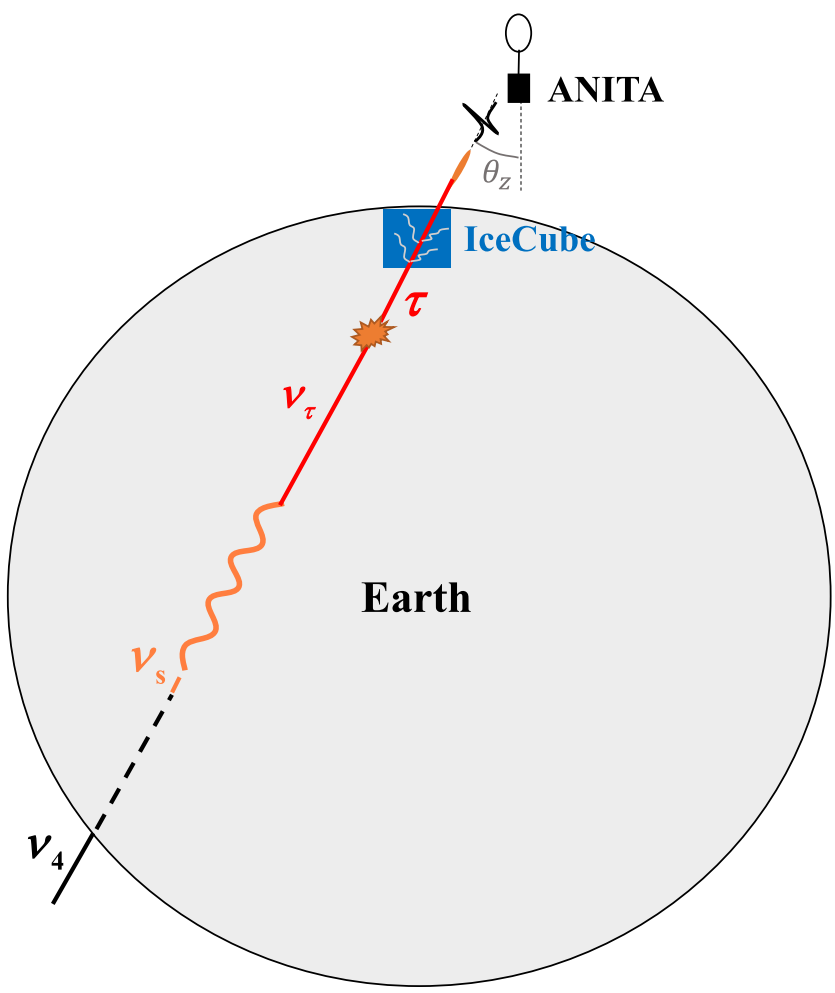

FIG. 1. A schematic diagram for the sterile neutrino explanation. The decay of a $\tau$ lepton above the Antarctic surface induces the radio pulse signal of ANITA. When the $\tau$ lepton traverses the IceCube volume, the through-going track event should be identified.

to the strong attenuation effect at $E=1 \mathrm{EeV}$, the active component in the quantum state can not survive for a distance longer than $L_{\text {atten }} \approx 200 \mathrm{~km}$. In other words, the neutrino state will collapse back into the sterile state very quickly such that the drift process of the parametric resonance is severely disturbed. No periodic structure in the Earth exists within the length scale $L_{\text {atten }} \approx 200 \mathrm{~km}$; therefore, the parametric resonance is not important in our case.

In Fig. 2, we show the evolution of the EeV neutrino fluxes with respect to the traveling distance in the Earth. With the zenith angle of $\theta_{z}=55^{\circ}$, the corresponding chord length through the Earth matter is around $7309 \mathrm{~km}$. The dashed curve demonstrates the attenuation effect for the standard active neutrinos. Reference [5] has given the Earth attenuation factor as $4 \times 10^{-6}$ for the event 3985267 . Our numerical results of the attenuation factor are $1.2 \times 10^{-9}$ for the event 3985267 , and $1.4 \times 10^{-13}$ for the event 15717147, with both CC and NC interactions taken into account ${ }^{4}$ The blue curves represent the survival probability

\footnotetext{
${ }^{4}$ As has been mentioned before, Ref. [5] has only considered the $\mathrm{CC}$ interaction in their estimation of the attenuation length. We have included the NC interaction for the conservative purpose. The actual attenuation factor should be larger after the regeneration effect is included.
} 


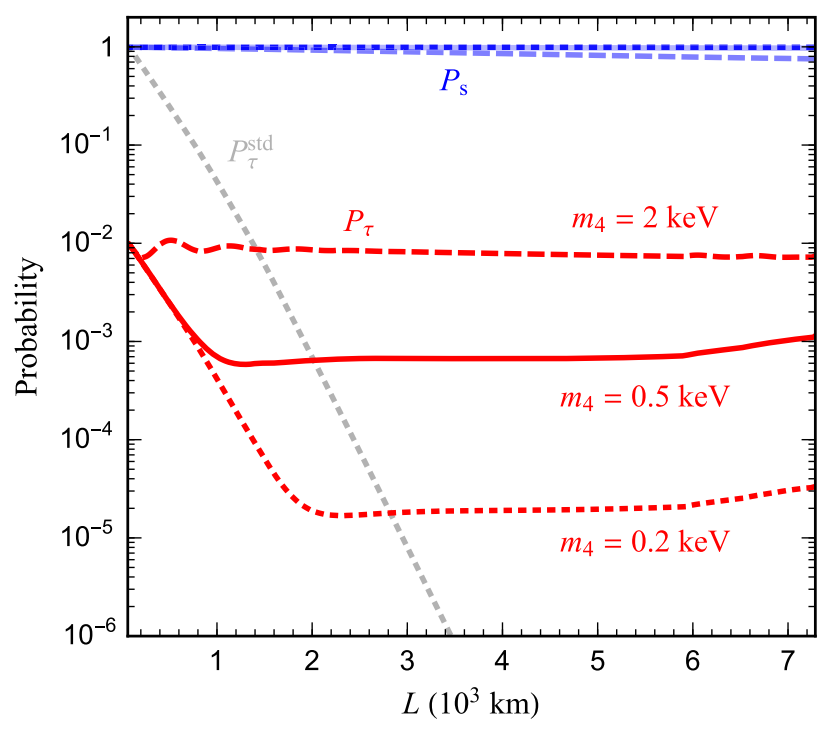

FIG. 2. The evolution of the EeV neutrino survival probability with respect to the traveling distance for the ANITA event 15717147. The active-sterile mixing angle is chosen as $\theta=0.1$, and the mass of $\nu_{4}$ can be $2 \mathrm{keV}$ (dashed curves), $0.5 \mathrm{keV}$ (solid curves), and $0.2 \mathrm{keV}$ (dotted curves). The dotted gray curve is the evolution of the standard $\nu_{\tau}$ flux. The blue curves stand for the survival probabilities of the sterile component $\nu_{\mathrm{s}}$, while the red curves stand for that of the $\nu_{\tau}$ component in the context of active-sterile mixing.

of the sterile neutrino component $\left|c_{\mathrm{s}}\right|^{2}$, and they stay almost around one during the propagation. The red curves stand for the evolutions of the $\nu_{\tau}$ fraction in $\nu_{4}(t)$. The survival probability of $\nu_{\tau}$ decreases with a smaller $\nu_{4}$ mass because of the increasing oscillation length. For $m_{4}=2 \mathrm{keV}$, the survival probability of $\nu_{\tau}$ fluctuates around 0.01 due to the continuous regeneration from $\nu_{\mathrm{s}}$ flux.

The two ANITA events both have energies around $0.6 \mathrm{EeV}$, i.e., $\quad E_{3985267}=0.6 \pm 0.4 \mathrm{EeV}, \quad E_{15717147}=$ $0.56_{-0.2}^{+0.3} \mathrm{EeV}$. To simplify our calculation, we assume that the initial $\nu_{4}$ flux has an almost monochromatic energy around $1 \mathrm{EeV}$. After these neutrinos enter the Earth, they can propagate almost freely to the other side of the Earth just as the $m_{4}=2 \mathrm{keV}$ case in Fig. 2. With a $\nu_{\tau}$ residue in the interaction region with a depth around tens of kilometer below the ANITA balloon, the flux is eventually transformed into observable $\tau$-lepton flux by the CC interaction. We define the efficiency of the initial $\nu_{4}$ particles being transformed into $\tau$ lepton as

$$
\epsilon(\Omega)=\frac{\mathrm{d} \Phi_{\tau}\left(E_{\min }, E_{\max }\right) / \mathrm{d} \Omega}{\mathrm{d} \Phi_{\nu_{4}}\left(E_{0}\right) / \mathrm{d} \Omega},
$$

where $E_{0}=1 \mathrm{EeV}$ is the initial neutrino energy, $\Phi_{\nu_{4}}$ stands for the isotropic flux of neutrinos, and $\Phi_{\tau}\left(E_{\min }, E_{\max }\right)$ is the produced $\tau$-lepton flux in the energy range of $\left[E_{\min }, E_{\max }\right]$ when they arrive at the Antarctic surface. We set $E_{\text {min }}=$ $0.2 \mathrm{EeV}$ and $E_{\max }=1 \mathrm{EeV}$ for our calculation. The transforming efficiency $\epsilon(\Omega)$ measures the fraction of neutrinos

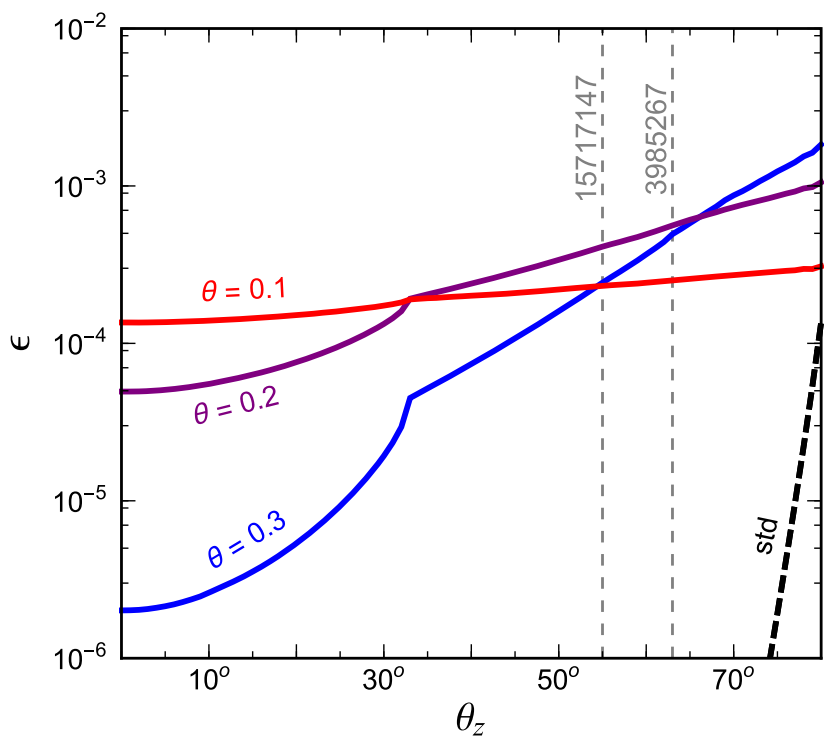

FIG. 3. The $\tau$ lepton transforming efficiency $\epsilon$ with respect to the emergence zenith angle. The sterile neutrino mass is fixed as $m_{4}=2 \mathrm{keV}$. The red, purple, and blue curves stand for the cases with mixing angles of $\theta=0.1, \theta=0.2$, and $\theta=0.3$, respectively. The dashed curve in the bottom-right corner is just the efficiency of the pure active neutrino flux scenario. The two vertical lines correspond to the emergence angles of the two ANITA events 15717147 and 3985267 . Note that $\theta_{z} \gtrsim 80^{\circ}$ is nearly above the ANITA horizon. The knee around $\theta_{z}=30^{\circ}$ is due to the big density jump between the Earth outer core and mantle. The energy loss of $\tau$ leptons are simulated with the ASW model $[46,47]$.

being converted into the detectable $\tau$ leptons during their way to ANITA; it should be noted that $\epsilon(\Omega)$ is direction dependent. In Fig. 3, we show the angular dependence of the $\epsilon(\Omega)$ for different cases with $m_{4}=2 \mathrm{keV}$. The dashed curve in the bottom-right corner shows the efficiency in the standard scenario. The $\tau$ leptons induced by the standard isotropic active neutrino flux should be distributed around the large zenith angles; therefore, the Earth-skimming shower should dominate the events as has been expected. The red curve with active-sterile mixing angle of $\theta=0.1$ is almost uniformly distributed for the entire zenith angle range. The efficiency is around $10^{-4}$, which means that no matter which angle the neutrino flux comes from, there will always be one observable $\tau$ lepton emitted after $10^{4} \nu_{4}$ neutrinos enter the Earth. The Earth-skimming events do not have too much advantage over the steep events in this case. However, as the active-sterile mixing angle increases, the sterile neutrino would become not so sterile due to the large mixing with the active neutrino. The efficiency tends to converge into the standard case.

\section{ANITA EVENTS ESTIMATION}

Because of the small pulse angle of the EeV $\tau$-decay shower, only a very small fraction of the $\tau$-lepton flux obtained in the last section can be captured by the antennas of 
ANITA. The angle of the radio cone should be around $1^{\circ}[8]$, so we expect the geometric area of ANITA should be about $A_{\mathrm{gm}} \approx 2 \pi\left(D \times 1^{\circ}\right)^{2}$ with $D$ being the distance from the ANITA payload to the initial point of the shower. For event 15717147 with $\theta_{z}=55^{\circ}$, we obtain the geometric area as $\sim 7.5 \mathrm{~km}^{2}$, slightly larger than the estimation of the ANITA group $\sim 4 \mathrm{~km}^{2}$ [8]. The realistic geometric area estimation requires the dedicated Monte Carlo simulation; here we simply assume a constant geometric area of $4 \mathrm{~km}^{2}$ for all emergence angles to proceed our estimation. Simulations show that the $\tau$-lepton decay shower from a larger zenith angle would have smaller impulse power [48]; thus, the steep shower seems more likely to be detected by ANITA than the Earth-skimming shower in the realistic case. The flux of the $\mathrm{EeV} \nu_{4}$ is bounded by the IceCube observation as $\mathrm{d} \Phi_{\nu_{4}} / \mathrm{d} \Omega \lesssim 2 \times 10^{-15}[0.1 / \sin \theta]^{2} \mathrm{~cm}^{-2} \mathrm{~s}^{-1} \mathrm{sr}^{-1}$; note that the flux limit is relaxed by the mixing angle suppression. The final event is obtained as

$\mathcal{E}_{\mathrm{ANITA}}=\int \mathrm{d} \Omega \cdot \frac{\mathrm{d} \Phi_{\nu_{4}}}{\mathrm{~d} \Omega} \times \epsilon(\Omega) \times A_{\mathrm{gm}}^{\mathrm{ANITA}}(\Omega) \times T_{\mathrm{ANITA}} \approx 0.9$,

where $A_{\mathrm{gm}}^{\mathrm{ANITA}}(\Omega)$ is simply fixed to $4 \mathrm{~km}^{2}$ as mentioned before, $T_{\text {ANITA }}$ is the three months of exposure for ANITA, $\epsilon(\Omega)$ is obtained with $m_{4}=2 \mathrm{keV}, \theta=0.1$ in the last section, and the flux takes the saturated value of the IceCube bound $\mathrm{d} \Phi_{\nu_{4}} / \mathrm{d} \Omega=2 \times 10^{-15}[0.1 / \sin \theta]^{2} \mathrm{~cm}^{-2}$ $\mathrm{s}^{-1} \mathrm{sr}^{-1}$. One can identify the effective area as $A_{\text {eff }}(\Omega)=$ $\epsilon(\Omega) A_{\mathrm{gm}}^{\mathrm{ANITA}}(\Omega) \approx 10^{7} \mathrm{~cm}^{2}$, much smaller than the estimation $A_{\text {eff }} \approx 10^{11} \mathrm{~cm}^{2}$ of Ref. [10]. An event number of 0.9 is obviously consistent with the ANITA observation. Let us check the situation for other experiments. As has been shown in Fig. 1, the upgoing $\tau$ lepton should unavoidably induce the Cherenkov light in the IceCube detector. The IceCube has a geometric area averagely around $1 \mathrm{~km}^{25}$ for the through-going track events; therefore, we can estimate the event number of IceCube with six years observation as $\mathcal{E}_{\mathrm{IC}}=6$, using $A_{\mathrm{gm}}^{\mathrm{IC}}(\Omega) \approx 1 \mathrm{~km}^{2}, T_{\mathrm{IC}} \approx 6$ years. As has been pointed out in [11,49], IceCube might already have observed one such $\tau$-track event with an energy $\gtrsim 0.1 \mathrm{EeV}$ and emergence angle of $11.5^{\circ}$ below the horizon. The deposited energy of this track is $(2.6 \pm 0.3) \mathrm{PeV}$ [50], implying a $\mu$-lepton track with an energy $\gtrsim 10 \mathrm{PeV}$ or a $\tau$-lepton track with an energy $\gtrsim 0.1 \mathrm{EeV}$. No matter whether this event is an $\mathrm{EeV} \tau$-lepton track or not, the IceCube observation is in considerable tension with ANITA under the sterile hypothesis. The nonobservation

\footnotetext{
${ }^{5}$ The geometric structure of the IceCube volume with detectors is like a hexagonal prism with the base area around $0.85 \mathrm{~km}^{2}$ and vertical length of $1 \mathrm{~km}$. If we assume the event acceptance of a $\tau$ lepton is $100 \%$ once it passes through the detector volume, the geometric area for the upgoing event should be between $0.85 \mathrm{~km}^{2}$ and $1.2 \mathrm{~km}^{2}$ for different upgoing zenith angles.
}

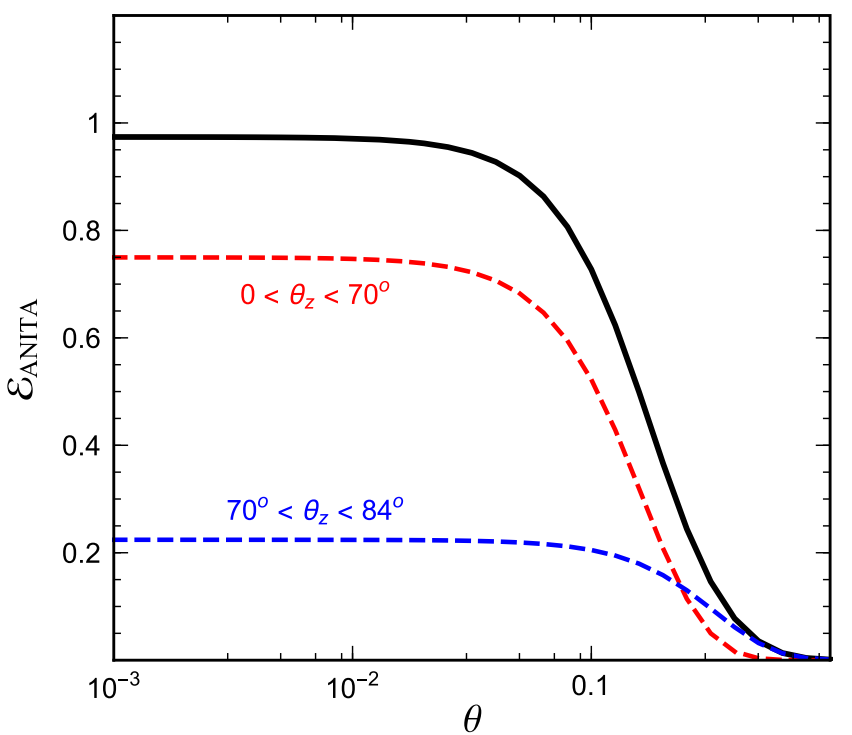

FIG. 4. The event number of ANITA for three months of exposure. For each mixing angle $\theta$, the corresponding IceCube bound on a $\nu_{4}$ flux is saturated. The black solid curve is the event number for the total sky. The red (blue) dashed curve stands for the shower events emitted from $>20^{\circ}\left(<20^{\circ}\right)$ below the horizontal. $\theta_{z}=84^{\circ}$ is the zenith angle of the ANITA horizon.

of EeV neutrino events at AUGER should not be a problem with a viewing angle of only a few degrees below the horizon for the Earth-skimming events [51].

In Fig. 4, we plot the relation between the ANITA's event number with three months of observation and the activesterile mixing angles. The mass of $\nu_{4}$ is chosen to be $2 \mathrm{keV}$, and the results do not differ much for $m_{4}>2 \mathrm{keV}$. The solid black curve is the total event number. The red dashed curve is the events within the zenith angle range of $\left[0^{\circ}, 70^{\circ}\right]$, which is larger than the blue one, i.e., the events within the zenith angle range of $\left[70^{\circ}, 84^{\circ}\right]$ for $\theta \lesssim 0.1$. Note that the event number curves in Fig. 4 can be extrapolated towards very small mixing angles, but the associated sterile neutrino flux is also required to be stronger. The flux should stay around the IceCube upper limit $\mathrm{d} \Phi_{\nu_{4}} / \mathrm{d} \Omega \approx 2 \times 10^{-15}$ $[0.1 / \sin \theta]^{2} \mathrm{~cm}^{-2} \mathrm{~s}^{-1} \mathrm{sr}^{-1}$. A more reliable result depending on the dedicated Monte Carlo simulations of the ANITA experiment is beyond the scope of the present work.

\section{CONCLUSION}

In this work, we have reinvestigated the possibility of using sterile neutrino origin to explain the ANITA anomalous events. We find that the quantum decoherence effect is very important to account for the propagation behavior of an EeV neutrino flux. The $\nu_{\tau}$ flux can be regenerated by the oscillation of $\nu_{\mathrm{S}}$ state during their propagation inside the Earth. For the sterile neutrinos with $m_{4} \gtrsim 1 \mathrm{keV}$, the Earth is almost transparent to the $\nu_{\tau}$ component in the $\nu_{4}$ flux. In this way, the neutrinos can losslessly reach the interaction volume below the ANITA 
payload with very steep angles. We have estimated the ANITA and IceCube event number and find that on average the ANITA experiment is able to observe one event during the three months of exposure, while the IceCube is supposed to detect six events for its six years of data taking. To resolve the ANITA anomaly itself, the flux of $\nu_{4}$ should be around the upper IceCube limit $\mathrm{d} \Phi_{\nu_{4}} / \mathrm{d} \Omega \approx 2 \times 10^{-15}[0.1 / \sin \theta]^{2} \mathrm{~cm}^{-2} \mathrm{~s}^{-1} \mathrm{sr}^{-1}$. We have scanned the whole sterile neutrino parameter space and find that the requirements on the sterile neutrino parameters are $m_{4} \gtrsim 1 \mathrm{keV}, \theta \lesssim 0.1$. If the dark matter decay is the source of the sterile neutrinos, the mixing angle should not be too small, because it might be too difficult for the dark matter decay to produce so much strong neutrino flux that it saturates the IceCube bound. In our framework, the predicted $\mathrm{EeV} \tau$-lepton track event number of IceCube is averagely 6 times of the $\tau$-lepton decay shower event number of ANITA, i.e., $\mathcal{E}_{\text {IC }} / \mathcal{E}_{\text {ANITA }} \approx 6$, and this result is independent of the sterile neutrino parameters. Even though there is an $\mathcal{O}(0.1 \mathrm{EeV})$ track candidate for the IceCube observation, these two experiments together are in strong tension with the sterile neutrino explanation. However, we expect the dedicated ANITA simulation to draw a more solid conclusion.

\section{ACKNOWLEDGMENTS}

I am indebted to S. Zhou for suggesting this work and for many valuable discussions and suggestions. I am also grateful to N. Nath, Q. R. Liu, and J. J. Liao for useful discussions. This work is supported by the National Natural Science Foundation of China under Grant No. 11775232.
[1] K. Greisen, End to the Cosmic Ray Spectrum?, Phys. Rev. Lett. 16, 748 (1966).

[2] G. T. Zatsepin and V.A. Kuzmin, Upper limit of the spectrum of cosmic rays, Pis'ma Zh. Eksp. Teor. Fiz. 4, 114 (1966) [JETP Lett. 4, 78 (1966)].

[3] V.S. Berezinsky and G. T. Zatsepin, Cosmic rays at ultrahigh-energies (neutrino?), Phys. Lett. 28B, 423 (1969).

[4] P. W. Gorham et al. (ANITA Collaboration), The Antarctic impulsive transient antenna ultra-high energy neutrino detector design, performance, and sensitivity for 2006-2007 balloon flight, Astropart. Phys. 32, 10 (2009).

[5] P. W. Gorham et al., Characteristics of Four Upwardpointing Cosmic-ray-like Events Observed with ANITA, Phys. Rev. Lett. 117, 071101 (2016).

[6] M. G. Aartsen et al. (IceCube Collaboration), Differential limit on the extremely-high-energy cosmic neutrino flux in the presence of astrophysical background from nine years of IceCube data, arXiv:1807.01820.

[7] M. G. Aartsen et al. (IceCube Collaboration), The IceCube neutrino observatory-contributions to ICRC 2017 Part II: Properties of the atmospheric and astrophysical neutrino flux, arXiv:1710.01191.

[8] P. W. Gorham et al. (ANITA Collaboration), Observation of an unusual upward-going cosmic-ray-like event in the third flight of ANITA, arXiv:1803.05088.

[9] P. Motloch, J. Alvarez-Muiz, P. Privitera, and E. Zas, Can transition radiation explain the ANITA event 3985267?, Phys. Rev. D 95, 043004 (2017).

[10] J. F. Cherry and I. M. Shoemaker, A sterile neutrino origin for the upward directed cosmic ray shower detected by ANITA, arXiv:1802.01611.

[11] L. A. Anchordoqui, V. Barger, J. G. Learned, D. Marfatia, and T. J. Weiler, Upgoing ANITA events as evidence of the $C P T$ symmetric universe, J. High Energy Phys. 01 (2018) 13.
[12] P. Minkowski, $\mu \rightarrow e \gamma$ at a rate of one out of $10^{9}$ muon decays?, Phys. Lett. 67B, 421 (1977).

[13] T. Yanagida, Horizontal symmetry and masses of neutrinos, Conf. Proc. C 7902131, 95 (1979).

[14] S. L. Glashow, The future of elementary particle physics, NATO Sci. Ser. B 61, 687 (1980).

[15] M. Gell-Mann, P. Ramond, and R. Slansky, Complex spinors and unified theories, Conf. Proc. C 790927, 315 (1979).

[16] M. Drewes et al., A white paper on keV sterile neutrino dark matter, J. Cosmol. Astropart. Phys. 01 (2017) 025.

[17] A. Aguilar-Arevalo et al. (LSND Collaboration), Evidence for neutrino oscillations from the observation of antineutrino(electron) appearance in a anti-neutrino(muon) beam, Phys. Rev. D 64, 112007 (2001).

[18] A. A. Aguilar-Arevalo et al. (MiniBooNE Collaboration), Unexplained Excess of Electron-Like Events From a 1-GeV Neutrino Beam, Phys. Rev. Lett. 102, 101802 (2009).

[19] C. Giunti and M. Laveder, Statistical significance of the gallium anomaly, Phys. Rev. C 83, 065504 (2011).

[20] G. Mention, M. Fechner, T. Lasserre, T. A. Mueller, D. Lhuillier, M. Cribier, and A. Letourneau, The reactor antineutrino anomaly, Phys. Rev. D 83, 073006 (2011).

[21] J. N. Abdurashitov et al. (SAGE Collaboration), Measurement of the solar neutrino capture rate with gallium metal. III: Results for the 2002-2007 data-taking period, Phys. Rev. C 80, 015807 (2009).

[22] F. Kaether, W. Hampel, G. Heusser, J. Kiko, and T. Kirsten, Reanalysis of the GALLEX solar neutrino flux and source experiments, Phys. Lett. B 685, 47 (2010).

[23] M. G. Aartsen et al. (IceCube Collaboration), Search for neutrinos from decaying dark matter with IceCube, arXiv: 1804.03848 .

[24] Y. Ema, R. Jinno, and T. Moroi, Cosmic-ray neutrinos from the decay of long-lived particle and the recent IceCube result, Phys. Lett. B 733, 120 (2014). 
[25] A. Esmaili and P.D. Serpico, Are IceCube neutrinos unveiling PeV-scale decaying dark matter?, J. Cosmol. Astropart. Phys. 11 (2013) 054.

[26] B. Feldstein, A. Kusenko, S. Matsumoto, and T. T. Yanagida, Neutrinos at IceCube from heavy decaying dark matter, Phys. Rev. D 88, 015004 (2013).

[27] P. Ko and Y. Tang, IceCube events from heavy DM decays through the right-handed neutrino portal, Phys. Lett. B 751, 81 (2015).

[28] M. Kachelriess, The rise and fall of top-down models as main UHECR sources, arXiv:0810.3017.

[29] P. S. B. Dev, D. Kazanas, R. N. Mohapatra, V. L. Teplitz, and Y. Zhang, Heavy right-handed neutrino dark matter and $\mathrm{PeV}$ neutrinos at IceCube, J. Cosmol. Astropart. Phys. 08 (2016) 034.

[30] Y. Sui and P. S. B. Dev, A combined astrophysical and dark matter interpretation of the IceCube HESE and throughgoing muon events, J. Cosmol. Astropart. Phys. 07 (2018) 020.

[31] M. Kachelriess, O.E. Kalashev, and M. Y. Kuznetsov, Heavy decaying dark matter and IceCube high energy neutrinos, arXiv:1805.04500.

[32] J. F. Cherry, A. Friedland, and I. M. Shoemaker, Neutrino portal dark matter: From dwarf galaxies to IceCube, arXiv: 1411.1071

[33] J. F. Cherry, A. Friedland, and I. M. Shoemaker, Shortbaseline neutrino oscillations, Planck, and IceCube, arXiv: 1605.06506

[34] B. Ahlgren, T. Ohlsson, and S. Zhou, Comment on Is Dark Matter with Long-Range Interactions a Solution to All Small-Scale Problems of Cold Dark Matter Cosmology?, Phys. Rev. Lett. 111, 199001 (2013).

[35] G. y. Huang, T. Ohlsson, and S. Zhou, Observational constraints on secret neutrino interactions from big bang nucleosynthesis, Phys. Rev. D 97, 075009 (2018).

[36] Y. Farzan, M. Lindner, W. Rodejohann, and X. J. Xu, Probing neutrino coupling to a light scalar with coherent neutrino scattering, J. High Energy Phys. 05 (2018) 066.

[37] Y. S. Jeong, S. Palomares-Ruiz, M. H. Reno, and I. Sarcevic, Probing secret interactions of $\mathrm{eV}$-scale sterile neutrinos with the diffuse supernova neutrino background, J. Cosmol. Astropart. Phys. 06 (2018) 019.

[38] J. M. Berryman, V. Brdar, and P. Huber, Nuclear and particle conspiracy solves both reactor antineutrino anomalies, arXiv:1803.08506.
[39] M. Blennow, E. Fernandez-Martinez, J. Gehrlein, J. Hernandez-Garcia, and J. Salvado, IceCube bounds on sterile neutrinos above $10 \mathrm{eV}$, arXiv:1803.02362.

[40] R. A. Harris and L. Stodolsky, Two state systems in media and "Turing's paradox", Phys. Lett. 116B, 464 (1982).

[41] L. Stodolsky, On the treatment of neutrino oscillations in a thermal environment, Phys. Rev. D 36, 2273 (1987).

[42] G. Raffelt, G. Sigl, and L. Stodolsky, Non-Abelian Boltzmann Equation for Mixing and Decoherence, Phys. Rev. Lett. 70, 2363 (1993); Erratum, Phys. Rev. Lett.98, 069902 (E) (2007).

[43] G. y. Huang, J. H. Liu, and S. Zhou, Matter effects on the flavor conversions of solar neutrinos and highenergy astrophysical neutrinos, Nucl. Phys. B931, 324 (2018).

[44] A. M. Dziewonski and D. L. Anderson, Preliminary reference earth model, Phys. Earth Planet. Inter. 25, 297 (1981).

[45] R. Gandhi, C. Quigg, M. H. Reno, and I. Sarcevic, Ultrahigh-energy neutrino interactions, Astropart. Phys. 5, 81 (1996).

[46] N. Armesto, C. A. Salgado, and U. A. Wiedemann, Relating High-Energy Lepton-Hadron, Proton-Nucleus and NucleusNucleus Collisions through Geometric Scaling, Phys. Rev. Lett. 94, 022002 (2005).

[47] J. Alvarez-Muiz, W. R. Carvalho, K. Payet, A. RomeroWolf, H. Schoorlemmer, and E. Zas, Comprehensive approach to tau-lepton production by high-energy tau neutrinos propagating through the Earth, Phys. Rev. D 97, 023021 (2018).

[48] A. Romero-Wolf (ANITA Collaboration), Upward-pointing cosmic-ray-like events observed with ANITA, Proc. Sci., ICRC2017 (2017) 935.

[49] M. D. Kistler and R. Laha, Multi-PeV Signals from a New astrophysical Neutrino Flux beyond the Glashow Resonance, Phys. Rev. Lett. 120, 241105 (2018).

[50] M. G. Aartsen et al. (IceCube Collaboration), Observation and characterization of a cosmic muon neutrino flux from the northern hemisphere using six years of IceCube data, Astrophys. J. 833, 3 (2016).

[51] A. Aab et al. (Pierre Auger Collaboration), Improved limit to the diffuse flux of ultrahigh energy neutrinos from the Pierre Auger Observatory, Phys. Rev. D 91, 092008 (2015). 